\title{
COHESIVE SETS: COUNTABLE AND UNCOUNTABLE
}

\author{
RICHARD A. SHORE ${ }^{1}$
}

\begin{abstract}
We show that many uncountable admissible ordinals (including some cardinals) as well as all countable admissible ordinals have cohesive subsets. Exactly which cardinals have cohesive subsets, however, is shown to depend on set-theoretic assumptions such as $V=L$ or a large cardinal axiom.
\end{abstract}

The study of recursion theory on the ordinals was initiated by Takeuti and then generalized by several others to all admissible ordinals. The analogy with ordinary recursion theory has been quite striking and particularly so for the theory of degrees of unsolvability. Many major theorems, especially ones about recursively enumerable degrees have been successfully generalized to all admissible ordinals. Thus for example, Sacks and Simpson [3] have introduced the finite injury priority argument into $\alpha$-recursion theory to construct two incomparable $\alpha$-r.e. degrees. Indeed even an infinite injury argument has been successfully adapted to this general setting to prove that the $\alpha$-r.e. degrees are dense for every admissible $\alpha$ [5]. In general it seems fair to say that although the proofs are often somewhat different and usually more complicated than those in ordinary recursion theory, the theorems about degrees (at least the r.e. ones) seem to carry over.

The situation changes drastically when one turns from degrees to sets even if one restricts one's attention to the recursively enumerable sets. Of course the phenomena of nonregularity poses many interesting problems along these lines [4], [7] but a more striking example is provided by the notion of maximal set. (An $\alpha$-r.e. set is maximal if and only if its complement is $\alpha$-infinite but cannot be split into two $\alpha$-infinite pieces by an $\alpha$-r.e. set.) It is a well-known theorem of Friedberg that such sets exist in ordinary recursion theory [2]. On the other hand, Lerman and Simpson [1] have shown that there are no maximal r.e. sets for any uncountable admissible.

Finally, if we drop the requirement of recursive enumerability, the situation becomes even further removed from that of ordinary recursion

Received by the editors December 11, 1972.

AMS (MOS) subject classifications (1970). Primary 02F27; Secondary 02K05.

Key words and phrases. Admissible ordinal, cohesive set.

${ }^{1}$ During the preparation of this paper the author was partially supported by NSF Grant GP-28700.

(c) American Mathematical Society 1974 
theory. Thus, Lerman and Simpson [1] ask if there are, for example, any uncountable cardinals $\alpha$ with cohesive subsets. (An $\alpha$-infinite set is cohesive if and only if it cannot be split into two $\alpha$-infinite pieces by any $\alpha$-r.e. set.) We here answer this question affirmatively. However, their general question as to which admissibles have cohesive subsets turns out to be more complicated. The methods of [1] show, for example, that if $V=L$ then $\boldsymbol{\aleph}_{1}$ (like each other successor cardinal) has no cohesive set. On the other hand, we will see that a large cardinal assumption implies that $\omega_{1}^{L}$ as well as $\boldsymbol{\aleph}_{1}$ (indeed all true cardinals) have cohesive sets. Thus we have an example of a recursion theoretic question about sets which in general is not even absolute. Appropriately enough our methods of proof are (with the addition of definability considerations) much like those associated with Erdös' style partition relations and other large cardinal arguments.

We now turn to the theorems.

THEOREM 1. If $L_{\alpha}$ is $\Sigma_{3}$-admissible, cofinal with $\omega$ and satisfies the power set axiom, then there is a cohesive subset of $\alpha$.

Proof. We inductively build a tree $\left(T,<_{T}\right)$ whose nodes $x$ are members of $\alpha$ and have associated with them sets $E_{x} \subseteq \alpha$, consisting of all successors of $x$ in $T$. To begin, we let 0 be the unique node of rank 0 and set $E_{0}=\alpha-\{0\}$. At each level $\beta<\alpha$ we consider every node $x$ of rank $\beta$ and split $E_{x}$ into two pieces, $E_{x} \cap R_{\beta}$ and $E_{x}-R_{\beta}$. $\left(R_{\beta}\right.$ is the $\beta$ th $\alpha$-r.e. set.) We then appoint the least elements, $y_{1}$ and $y_{2}$, of these sets (if they are nonempty) as the immediate successors of $x$ (of rank $\beta+1$ ). We also set $E_{y_{1}}=\left(E_{x} \cap R_{\beta}\right)-\left\{y_{1}\right\}$ and $E_{y_{2}}=\left(E_{x}-R_{\beta}\right)-\left\{y_{2}\right\}$. At limit levels $\lambda$ we naturally take all chains $\left\{x_{i}\right\}_{i<\lambda}$ in $L_{\alpha}$ and set the least element $y$ of $\bigcap_{i<\lambda} E_{x_{i}}$ (if there is one) as the successor (of rank $\lambda$ ) of $\left\{x_{i}\right\}_{i<\lambda}$ and put $E_{y}=$ $\bigcap_{i<\lambda} E_{x_{i}}-\{y\}$. We let $T_{\beta}^{x}$ denote the successors of $x$ of rank $\beta$.

The crucial fact about this tree is that every node $x$ such that $E_{x}$ is unbounded in $\alpha$ has successors $y$ at every level of the tree less than $\alpha$ such that $E_{y}$ is also unbounded in $\alpha$. Of course every $x \in \alpha$ is on this tree. Next we note that $T_{\beta}^{x}$ is the image of $\left(2^{\beta}\right)^{L_{\alpha}}$ under a partial $\Sigma_{2}$ function and so, by our assumptions, a member of $L_{\alpha}$. We then note that the partial map defined on $T_{\beta}^{x}$ by sending $z$ to $\bigcup E_{z}$ if $\bigcup E_{z}<\alpha$ is $\Sigma_{3}$. Thus if for some $\beta<\alpha$ there were no elements of $T_{\beta}^{x}$ as required we could map $T_{\beta}^{x}$ unboundedly into $\alpha$ by a $\Sigma_{3}$ function, contradicting the $\Sigma_{3}$ admissibility of $\alpha$. To verify these assertions, first define $F:\left(2^{\beta}\right)^{L_{\alpha} \rightarrow T_{\beta}^{x}}$ by $F(a)=y$ if and only if $y \in T_{\beta}^{x}$ and $(\forall \delta<\beta)\left(y \in R_{\delta} \leftrightarrow \delta \in a\right)$. It now suffices to show that $y \in T_{\beta}^{x}$ is a $\Sigma_{2}$ predicate. Say rank $x=\gamma$ and note that $y \in T_{\beta}^{x} \equiv x<_{T} y$ and rank $y=\beta \equiv x<y \&(\forall \delta<\gamma)\left(x \in R_{\delta} \leftrightarrow y \in R_{\delta}\right) \&(\exists f: \beta \rightarrow y)(f$ is one-one 
and order preserving and $\left.(\forall \eta<\beta)(\forall \delta<\eta)\left(f(\eta) \in R_{\delta} \leftrightarrow y \in R_{\delta}\right)\right) \& \sim$ $(\exists f: \beta+1 \rightarrow y)(f$ is one-one and order preserving and $(\forall \eta<\beta+1)$ $\left.(\forall \delta<\eta)\left(f(\eta) \in R_{\delta} \leftrightarrow y \in R_{\delta}\right)\right)$. Finally, the function taking $y$ of rank $\beta$ to $\bigcup E_{y}$ is the $\Sigma_{3}$-uniformization of the $I_{2}$-relation $S(y, z)$ given by $(\forall w)\left(y \leqq_{T} w \rightarrow w \in z\right) \equiv(\forall w)\left(y \in w \&(\forall \delta<\beta)\left(w \in R_{\delta} \leftrightarrow y \in R_{\delta}\right) \rightarrow w \in z\right)$.

Armed with this fact we can now build a path of length $\alpha$ through $T$ in $\omega$ many steps. Let $\left\{\beta_{i}\right\}_{i<\omega}$ be cofinal in $\alpha$. Begin with $x_{0}=0$ and so of course $E_{x_{0}}$ is unbounded in $\alpha$. We can now successively choose $x_{i}$ of rank $\beta_{i}$ such that $E_{x_{i}}$ is unbounded in $\alpha$. Clearly, $\left\{x_{i}\right\}_{i<\omega}$ traces out a path $P$ of length $\alpha$ in $T$. Moreover, $P=\left\{x \mid(\exists i<\omega)\left(x<_{T} x_{i}\right)\right\}$ is our desired cohesive set. For if we consider any $\alpha$-r.e. set $R_{\gamma}$ we see that either all elements of $P$ of rank $>\gamma$ fall into $R_{\gamma}$ or out of $R_{\gamma}$. As the elements of $P$ of rank $\leqq \gamma$ form a bounded $\Sigma_{2}$ set, it is $\alpha$-finite, and so therefore are its intersections with $R_{\gamma}$ and the complement of $R_{\gamma}$.

To see that this theorem answers the question of [1] we shall show that there are $\Sigma_{3}$ admissible cardinals cofinal with $\omega$. We begin with a definable $\Sigma_{3}$-skolem function $F$ for $L$. Let $A_{0}=\varnothing, A_{i+1}=\left(\bigcup\left(F^{\prime \prime}\left[A_{i}\right] \cap O R D\right)\right)^{+}$ and $A=\bigcup_{i<\omega} A_{i}$. $A$ is clearly a cardinal with the desired properties.

Turning now to the more general question of which admissibles have cohesive subsets we note first that we can adapt the above proof to establish

THEOREM 2. Every countable admissible $\alpha$ has a cohesive subset.

Proof. Just arrange the $\alpha$-r.e. sets in an $\omega$-sequence $R_{i}$ and build a tree as above. We can then of course get a path $\left\{y_{i}\right\}_{i<\omega}$ in $T$ with $E_{y_{i}}$ unbounded in $\alpha$ just by following a path determined by this requirement. We now form our cohesive set $\left\{x_{i} \mid i<\omega\right\}$ by choosing from each $E_{y_{i}}$ an element $x_{i}$ greater than $\beta_{i}$ where $\left\{\beta_{i}\right\}_{i<\omega}$ is unbounded in $\alpha$. This set is clearly cohesive.

We next note that, of course, if $\alpha$ is weakly compact in $L$ the tree of Theorem 1 has an $\alpha$-path and so has a cohesive subset. (This fact was already mentioned in [1].) Now the existence of a Ramsey cardinal, for example, guarantees that every true cardinal is weakly compact in $L$ [6]. Since $\omega_{1}^{L}$ is then of course countable we see that a large cardinal assumption implies that $\omega_{1}^{L}$ as well as every real cardinal has a cohesive subset. On the other hand, the methods of [1] show that if $\aleph_{1}$ is a successor cardinal of $L$ it has no cohesive subset. Thus we have our independence result. (Of course, no large cardinals are needed just to get a model with $\omega_{1}^{L}$ countable.)

In [1], Lerman and Simpson also indicated that if $V=L$, no cardinal $\alpha$ with $\Sigma_{2}$-cofinality less than $\alpha$ has a cohesive subset. Our final theorem shows that this is best possible in the sense that there are (provably in 
ZF) cardinals of $L$ which are $\Sigma_{2}$ - but not $\Sigma_{3}$-regular which have cohesive subsets. It also shows that there are real and constructible cardinals of all cofinalities with cohesive subsets.

THEOREM 3. If $\alpha$ is a cardinal of $L$ and for some $\beta>\alpha, L_{\alpha}$ is an elementary substructure of $L_{\beta}$ with respect to $\Sigma_{2}$ formulas $\left(L_{\alpha}<_{2} L_{\beta}\right)$, then $\alpha$ has a cohesive subset and indeed a constructible one.

Proof. Let $\gamma$ be the cofinality (in $L$ ) of $\alpha$ and let $\left\{\beta_{i}\right\}_{i<\gamma}$ be an unbounded increasing sequence in $\alpha$. We build our cohesive set $\left\{x_{i}\right\}_{i<\gamma}$ by induction following the path traced by $\alpha$ itself. Say we have $\left\{x_{i}\right\}_{i<\lambda}$. Let $\delta=$ $\max \left\{\bigcup_{i<\lambda} x_{i}, \beta_{\lambda}\right\}$. Let $f: \delta \rightarrow 2$ be given by $f(\eta)=0$ if and only if $L_{\beta} \vDash \varphi_{\eta}(\alpha)$ where $\varphi_{\eta}$ is the formula defining $R_{\eta}$ over $L_{\alpha}$. As $\alpha$ is a cardinal of $L, f \in L_{\alpha}$. Moreover, since $L_{\alpha} \prec_{2} L_{\beta}$ we see that

$$
L_{\alpha} \vDash(\exists x>\delta)(\forall \eta<\delta)\left(\varphi_{\eta}(x) \leftrightarrow f(\eta)=0\right) .
$$

Let $x_{\lambda}$ be such an $x$. $\left\{x_{i} \mid i<\gamma\right\}$ is now easily seen to be a cohesive subset of $\alpha$.

To see that this theorem does indeed supply the desired example, consider a $\Sigma_{2}$-Skolem function $F$ for $L$. As before we set $A_{0}=\varnothing, A_{\beta+1}=$ $\cup\left(F^{\prime \prime}\left[A_{\beta}\right] \cap \mathrm{ORD}\right)^{+}$and $A_{\lambda}=\bigcup_{\beta<\lambda} A_{\beta}$. If this is carried out in $L$ (i.e. successor cardinal in the sense of $L$ is intended) then for each limit $\lambda, A_{\lambda}$ is $\Sigma_{2}$-admissible but not $\Sigma_{3}$-admissible and has a cohesive subset by the theorem $\left(L_{A_{\lambda}}<_{2} L_{A_{\lambda+\omega}}\right)$. On the other hand if we do the construction in the real world we get $A_{\lambda}$ to be a true cardinal with cofinality that of $\lambda$. Of course the theorem still assures us that $A_{\lambda}$ has a cohesive subset.

\section{BIBLIOGRAPHY}

1. M. Lerman and S. G. Simpson, Maximal sets in $\alpha$-recursion theory, Israel J. Math. 4 (1973), 236-247.

2. H. Rogers, Jr., Theory of recursive functions and effective computability, McGrawHill, New York, 1967. MR 37 \#61.

3. G. E. Sacks and S. G. Simpson, The $\alpha$-finite injury method, Ann. Math. Logic 4 (1972), 343-367.

4. R. A. Shore, On splitting an $\alpha$-recursively enumerable set (to appear).

5. - The $\alpha$-recursively enumerable degrees are dense (in preparation).

6. J. H. Silver, Some applications of model theory in set theory, Ann. Math. Logic 3 (1971), 45-110.

7. S. G. Simpson, Admissible ordinals and recursion theory, Ph.D. Thesis, M.I.T., 1971.

Department of Mathematics, University of Chicago, Chicago, Illinois 60637 